\title{
Prospects of Future HERA Measurements
}

\author{
Olaf Behnke \\ Uni Heidelberg, Physik. Institut \\ Philosophenweg 12, Heidelberg 69120 - Germany
}

\begin{abstract}
The HERA ep collider shut down on June 30, 2007, after 15 years of operation. This paper briefly discusses some of the prospects for the final measurements of $\mathrm{H} 1$ and ZEUS with the complete HERA data of $\sim 500 \mathrm{pb}^{-1}$ per experiment. The focus is on the expected improvements for the proton parton densities (quarks and gluons) by measurements of inclusive structure functions, jet production and heavy flavours. Further topics like $\alpha_{s}$ determinations can be found in [1].
\end{abstract}

\section{Introduction}

The HERA neutral and charged current inclusive structure function data provide the basis for the most accurate proton parton density function (PDF) determinations down to low values of $x \sim 0.0001$. Figure 1 shows the current status of the published PDFs from H1 and ZEUS together with the H1 datasets that were used for the 'H1 PDF 2000' fit.

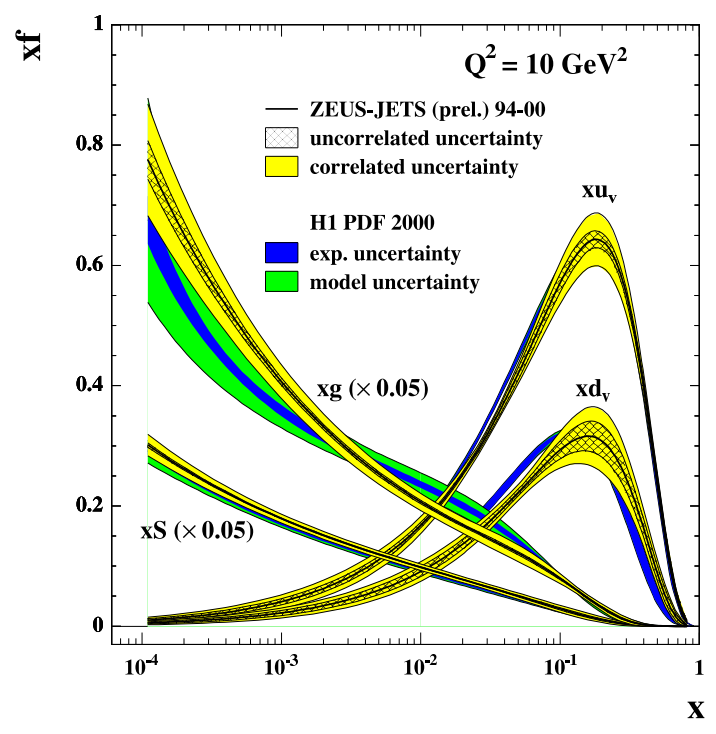

\begin{tabular}{|l|r|r|}
\hline $\begin{array}{c}\text { Datasets used for } \\
\mathrm{H} 1 \text { PDF } 2000\end{array}$ & $Q^{2}\left[\mathrm{GeV}^{2}\right]$ & $\mathrm{L}\left[\mathrm{pb}^{-1}\right]$ \\
\hline $\mathrm{NC} e^{+} p 96 / 97$ & $1.5-150$ & $2-20$ \\
\hline $\mathrm{NC} / \mathrm{CC} e^{+} p 94-00$ & $>150$ & 100 \\
\hline $\mathrm{NC} / \mathrm{CC} e^{-} p 94-00$ & $>150$ & 16 \\
\hline \hline $\begin{array}{c}\text { Additional datasets } \\
\text { for final H1 PDFs }\end{array}$ & $Q^{2}\left[\mathrm{GeV}^{2}\right]$ & $\mathrm{L}\left[\mathrm{pb}^{-1}\right]$ \\
\hline $\mathrm{NC}$ MB99/SVX00 & $0.2-8.5$ & $0.5-2$ \\
\hline $\mathrm{NC} e^{+} p 00$ & $10-150$ & 28 \\
\hline $\mathrm{NC} e^{ \pm} p 03 / 07$ & $\sim 30-150$ & 300 \\
$\mathrm{NC} / \mathrm{CC} e^{+} p 03 / 07$ & $>150$ & $\sim 180$ \\
\hline $\mathrm{NC} / \mathrm{CC} e^{-} p 03 / 07$ & $>150$ & $\sim 160$ \\
\hline DIS jets $94-07$ & $>150$ & $\sim 400$ \\
\hline$\gamma p$ dijets $94-07$ & 0 & $\sim 400$ \\
\hline
\end{tabular}

Figure 1: 'H1 PDF 2000' [2] and 'ZEUS JETS PDF' [3] parton distribution functions. The H1 datasets used for the 'H1 PDF 2000' are listed in the upper half of the table, the additional $\mathrm{H} 1$ data available for future PDF fits are given in the lower half.

The PDFs were obtained from QCD fits to the data in Next-to-Leading-Order (NLO) perturbative QCD. HERA provides unique information, especially on the gluon density. This can be exploited for predictions of other important processes such as Higgs production in gluon gluon fusion at the LHC. The visible differences between the H1 and ZEUS gluon 
HERA
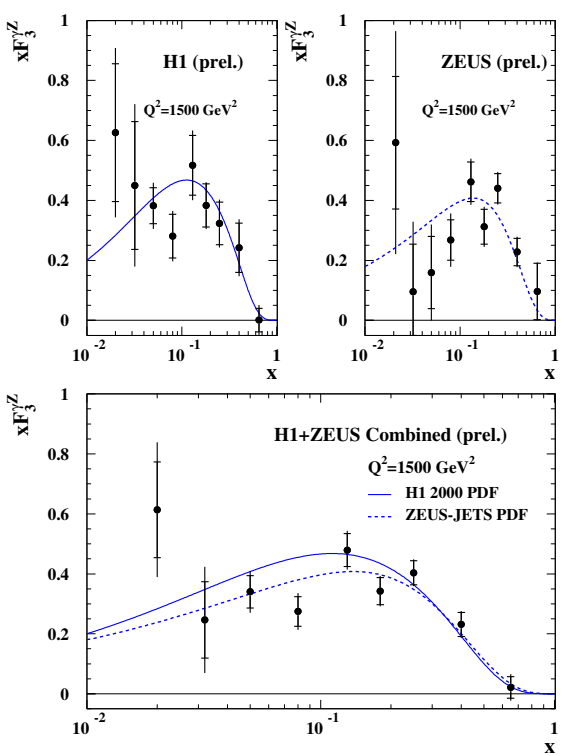

HERA I $\mathrm{e}^{+} \mathrm{p}$ Neutral Current Scattering - H1 and ZEUS

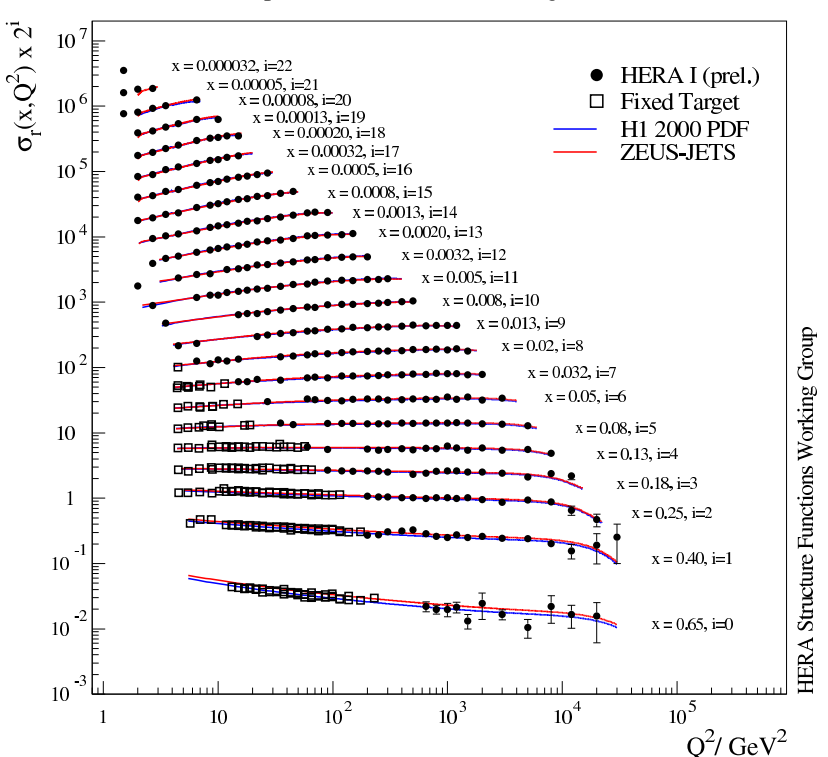

Figure 2: Combination of $\mathrm{H} 1$ and ZEUS measurements of the structure functions $x F_{3}[5]$ (left) and of the reduced NC cross section [6] (right).

densities (Fig. 1) indicate a seizable uncertainty which exceeds the one estimated from the error bands. One reason for this may be the different approaches used by H1 and ZEUS, e.g. for the functional form of the PDF parameterisations. In this paper we shall not investigate these or other model uncertainties but instead focus on the potential due to the inclusion of the new HERA data. The main $\mathrm{H} 1$ datasets available as input to improve the current PDFs are listed in the lower half of the table in Fig. 1. In some kinematic regions the statistics is improved by the new data by a factor of more than 10. This poses extreme challenges for the understanding of systematic uncertainties, especially at lower $Q^{2}$, where already the old data were mostly systematically limited. In the following some potential example improvements will be discussed that can be expected from these data. A more complete discussion can be found in [4].

\subsection{Valence quarks from $x F_{3}$}

$\mathrm{Z}$ exchange contributes to the neutral current reactions at high $Q^{2}$ at HERA. The sign of the interference term of photon and $\mathrm{Z}$ exchange depends on the charge of the lepton beam. This allows to access the valence quark distributions via the structure function $x F_{3}$ : $x F_{3} \propto \sigma_{N C}^{e^{+} p}-\sigma_{N C}^{e^{-} p} \propto 2 u_{v}+d_{v}$. The results from an H1 and ZEUS combined analysis [5] are shown in Fig. 2 (left). These measurements are based on about half of the full HERA dataset. They provide unique information on valence quarks at low $x<0.1$. The results are mostly limited by statistical errors, therefore the analysis of the complete HERA dataset will reduce the errors by approximately a factor of $\sqrt{2}$. 


\subsection{Sea quarks from $F_{2}$}

The main information on sea quarks is provided by the structure function $F_{2}$, which is dominating the inclusive $\mathrm{NC}$ cross sections at low $Q^{2}$ for not so large inelasticities $y$. The precision of the $F_{2}$ data that have been used to obtain the PDFs shown in Fig. 1 reaches $2-3 \%$ and is dominated by experimental systematic uncertainties. Improvements are at hand or can be expected due to:

- H1 and ZEUS combined [6] very recently the $\mathrm{NC}$ and $\mathrm{CC}$ inclusive cross section data which were used for their previous separate PDF determinations. The NC results are shown in Fig. 2 (right). The combination of the two datasets uses a method of weighted averaging, where parameters representing experimental systematic uncertainties (e.g. calorimeter energy scales) are also fitted. This leads to an effective cross calibration of the two experiments and finally to greatly reduced systematic errors. These H1 and ZEUS combined datasets will be used for a new PDF fit by the two collaborations.

- H1 plans to release soon the $28 \mathrm{pb}^{-1}$ of low and medium $Q^{2}$ inclusive $\mathrm{NC}$ data $(10<$ $Q^{2}<150 \mathrm{GeV}^{2}$ ) recorded in the year 2000. The main systematic errors are expected to be improved by up to a factor of two (e.g. the energy scale of the scattered electron) and thus an unprecedented precision of individual $F_{2}\left(x, Q^{2}\right)$ points of up to $1.5 \%$ is expected. The analysis of the $\sim 300 \mathrm{pb}^{-1}$ inclusive NC data from HERA II for $Q^{2}>30 \mathrm{GeV}^{2}$ needs to address the systematic errors at a new precision level and is expected to require a much longer timescale.

\subsection{Gluon density determinations}

At HERA the gluon density is mainly determined by the following four techniques:

- The statistically most precise determination is provided by the $F_{2}$ scaling violations, which at low $x$ can be approximated by $d F_{2} / d \ln \left(Q^{2}\right) \sim \alpha_{s}\left(Q^{2}\right) x g\left(x, Q^{2}\right)$. The improvements of the $F_{2}$ measurements discussed above will lead to similar improvements in the determination of the scaling violations and hence also of the gluon density.

- Jet production at lower $x$ is dominated by the the boson gluon fusion (BGF) process $\gamma^{*} g \rightarrow q \bar{q}$, where the photon is emitted by the beam lepton and thus provides direct access to the gluon density. ZEUS has already used high $p_{T}$ jets in photoproduction and jets in high $Q^{2}$ DIS events for the 'ZEUS Jets PDF' fit to improve the determination of the gluon density considerably, especially at high proton momentum fractions $x>0.01$. A nice feature at HERA is that the jet energy scale can be accurately calibrated via $p_{T}$ balance with the scattered electron. At the highest jet $p_{T}$ a scale precision of $\sim 1 \%$ has already been reached by ZEUS. For the largest $Q^{2}, x$ and/or $p_{T}$ the HERA jet data will thus remain statistically limited in contrast to jet data at the TEVATRON. A large improvement of the gluon density precision at $x>0.1$ of at least a factor of two can be expected from analysing the full HERA dataset and combining $\mathrm{H} 1$ and ZEUS measurements.

- Charm and beauty quarks are produced at HERA mainly in the BGF process $\gamma^{*} g \rightarrow c \bar{c}$ (or $b \bar{b}$ ) and thus also provide direct sensitivity to the proton gluon density. Charm events are tagged at HERA using fully reconstructed $D$ mesons or displaced track impact parameters, the latter exploiting the long lifetime of the charm quark. Compared to jets the identification of low $p_{T}$ charmed quarks provides access to gluons 
which carry small proton momentum fractions $x \leq 0.01$ at not so large factorisation scales. Theoretical analyses focus on the structure function $F_{2}^{c \bar{c}}$, which is defined as the part of $F_{2}$ due to events with charm quarks in the final state. H1 analyses [7] of the HERA I data based on the impact parameter method resulted in precisions for $F_{2}^{c \bar{c}}$ of $10-30 \%$ depending on $Q^{2}$. The new HERA II data sample provides a $\sim$ four times larger dataset. At lower $Q<60 \mathrm{GeV}^{2}$ total precisions of $5 \%$ should be obtainable for $F_{2}^{c \bar{c}}$. Similar uncertainties can be reached with the $D$ meson measurements. For the gluon density determination the charm data are statistically not competitive with the $F_{2}$ scaling violations. However they provide an important consistency check of the global QCD fits, especially for the highly non-trivial question of the charm quark mass treatment in the QCD analysis. Beauty production is probing the gluon density mainly at larger $x \sim 0.01$, where it is well known from the $F_{2}$ scaling violations. The $F_{2}^{b \bar{b}}$ measurements [7] by $\mathrm{H} 1$ based on displaced track impact parameters have reached a precision of 30-50\% for the HERA I data. Once again, improvements of a factor of 2 or more can be expected from the HERA II data. These measurements may provide constraints for the effective beauty quark density in the proton (neglecting the beauty quark mass) which can then be used to make predictions at very large scales at the LHC for processes such as $b \bar{b} \rightarrow \mathrm{H}$.

- The proton structure function $F_{L}$ provides another independent means to determine the gluon density. For a direct $F_{L}$ determination, HERA operated in the last 3 months of its lifetime with protons of lower energies than the nominal $E_{P}=920 \mathrm{GeV}$. H1 and ZEUS have each recorded about $13 \mathrm{pb}^{-1}$ and $7 \mathrm{pb}^{-1}$ of data at $E_{p}=460 \mathrm{GeV}$ and $E_{p}=575 \mathrm{GeV}$, respectively. These data may be used to measure $F_{L}$ with sufficient precision to separate between extreme gluon density parameterisations, especially for small $x<0.001$, where the gluon density is not so well known. These measurements cannot compete statistically with the determination from the $F_{2}$ scaling violations but provide (similarly as for charm) an important consistency test of the QCD models.

\section{Conclusion}

It has been demonstrated in this article that the analysis of the full HERA data sample will make significant progress in the determination of the quark and gluon densities in the proton, typically by a factor of two or more.

\section{References}

[1] Slides: http: //indico . cern. ch/contributionDisplay . py? contribId=253\&sessionId=10\&conf Id=9499

[2] C. Adloff et al. [H1 Collaboration], Eur. Phys. J. C 301 (2003) [hep-ex/0304003].

[3] S. Chekanov et al. [ZEUS Collaboration], Eur. Phys. J. C 421 (2005) [hep-ph/0503274].

[4] S. Alekhin et al., see page $78 \mathrm{ff}$, hep-ph/0601012.

[5] H1 and ZEUS Collaborations, contributed paper to ICHEP2006, Moscow 2006 http://www-h1.desy.de/h1/www/publications/htmlsplit/H1prelim-06-142.long.html.

[6] H1 and ZEUS Collaborations, contributed paper to LP2007, Daegu 2007 http://www-h1.desy.de/h1/www/publications/htmlsplit/H1prelim-07-141.long.html.

[7] A. Aktas et al. [H1 Collaboration], Eur. Phys. J. C 4523 (2006) [arXiv:hep-ex/0507081]. 